\title{
Design of PID controller for automatic voltage regulator and validation using hardware in the loop technique
}

\author{
Deepak M.Sajnekar ${ }^{b}$, Mohan L. Kolhe $^{\mathrm{a} *}$, S.B.Deshpande ${ }^{\mathrm{c}}$, R. M. Moharil ${ }^{\mathrm{b}}$, \\ Narayan P. Patidar ${ }^{\mathrm{d}}$, Koki Ogura ${ }^{\mathrm{e}}$, \\ ${ }^{a}$ Faculty of Engineering \&Science,University of Agder.,PO Box 422, Kristiansand, NO 4604, Norway. \\ ${ }^{b}$ Electrical Engineering Department, Yeshwantrao Chavan College of Engineering, Nagpur, Maharashtra,441110, India. \\ ${ }^{c}$ Electrical Engineering Department, Priyadarshini College of Engineering Nagpur, Maharashtra, 441110, India. \\ ${ }^{\mathrm{d}}$ Electrical Engineering Department, M.A. National Institute of Technology, Bhopal, M.P., 462007, India \\ ${ }^{e}$ Department of Electrical Engineering, Faculty of Science and Engineering, Kyushu Sangyo University, Fukuoka, Japan
}

\begin{abstract}
The digital excitation control system (DECS) used for brushless synchronous generators has recently become popular owing to its ease of operation and flexibility to manage the operating parameters to achieve good maintenance of the output terminal voltage at the rated value. The latest industrial digital automatic voltage regulator (AVR) is used in the forward path of DECS. This paper addresses the issues of the DECS that are responsible for deviating the terminal voltage from the rated value and consequently making the power system unstable. A solution to the issue is provided using a new method of tuning the PID controller that eases the impact of discretization on the terminal voltage and keeps the DECS compatible with industrial standards. The controller designed in this way will be useful to maintain the terminal voltage of the synchronous generator to enhance the stability of the power system to which many renewable energy sources are interconnected. The proposed method improves the performance of the excitation control system by reducing the overshoot from $75 \%$ to $16 \%$. The simulation results are presented using an industrial standard model of the excitation control system to demonstrate and compare the performance of the existing with the new digital excitation system. The newly designed controller was tested and validated using the "hardware in the loop" (HITL) technique.
\end{abstract}

Keywords: Automatic Voltage Regulator, PID Controller, Excitation Control System, Brushless Synchronous generator

\section{Introduction}

Synchronous generators excitation control systems are designed with controllers that allow a lot of setting flexibility to the operator to achieve an optimum generator terminal voltage response. Such controllers are still the choice of the industry owing to their high reliability, fast responses, simplicity and robustness [1]. However, owing to the advancements in "embedded system technology," the trend of upgrading the existing to a digital excitation control system (DECS) is in progress [2] that offers an easy setting flexibility to the operator. A comparison with the popular existing PID controller tuning method [3] explains the advantages and disadvantages of each method and implements the digital PID controller using the pole zero cancellation method.

The role of ECS in the optimum performance of the generator is very important in terms of maintaining the parameters of the controller at an adequate level, which ensures the dynamic behavior in the generator terminal voltage, reactive power, and the power factor to keep the system stable. The guidelines to the designer in regard the range of the performance indexes such as rise time, overshoot, settling time, gain margin, phase margin, and bandwidth of the controller used for the excitation control

* Manuscript received January 5, 2018; revised April 20, 2018.

Mohan L Kolhe. Tel.: +47 3723 3293; E-mail address: mohan.1.kolhe@uia.no

doi: $10.12720 /$ sgce.7.2.75-89 
system are provided in the documents [4-7].

A non-linear controller has been developed for the excitation system to ensure adequate dynamic behavior [8]. However, industries are using a linear controller for the excitation control system. They prefer control strategies that do not require the complete data of the plant. This is intended to avoid timeconsuming tests on the generator to evaluate the necessary parameters that are required to calculate the PID controller gains.

Currently, the developers of the control strategy use a complex model for the excitation control system that excludes the use of a standard model. The approach to use an intelligent control system provides a method to solve the stability problem of the system but is difficult to establish as a model using traditional equations. For example, the excitation control system is established using a neural network that is trained by the back-stepping algorithm, which improves the dynamic response in comparison to the conventional PID controller $[9,10]$. Moreover, precise data for the system is required to design a neural network to avoid a dangerous transient response at the initial point.

Combinations of the intelligent control algorithm, conventional PID and fractional order PID controller have been proposed [11], which uses the intelligent algorithm to tune the PID parameters. With the help of precise data for the system in all these cases, optimum performance is observed. However, in the absence of precise data, the initial requirements such as initial weight for the neural network, trails in ant colony algorithms [12], norms in multi-objective optimization, population size in swarm optimization [13] and rules in the fuzzy technique are not precisely set; therefore, the control process fails. To calculate the analog controller parameters for the excitation systems and to operate it within the protection limits, a method was proposed in [14] to make the performance indexes of the designed controller to comply with the IEEE standard but the method is only suitable for analog controllers.

The PID controller's self-tuning approach in modern excitation control system is used in the forward path. For example, the DECS reported in [15] uses the conventional method for PID gain calculation. Such a design results in an optimum performance even in the absence of precise data for the system. However, in this method the saturation data of the generator is used to calculate the PID gains and the sampling time of the processor needed to implement the digital PID controller is very small; this increases the labor and cost of the hardware. Feed forward control strategies have been evolved for further improvement of the performance in wind power applications [16], which show improved performance to achieve the rated speed when the wind speed is above the rated value. In some cases, a cascaded PD-PI and conventional PID controller have been considered for analyzing the dynamic response of the two-area interconnected power system in which to achieve enhanced performance controllers that are efficiently tuned by the "teaching learning based optimization" technique and improved performance has been reported [17]. The solution to the multi-machine low frequency inter-area oscillation is given by presenting an algorithm based wide area power system stabilizer [18], an unwanted effect caused due to sampling of error signal on the performance of the PID controller is in [19]. To minimize the uncertainties in the stability of the improved power system, a wide area fuzzy-2 logic-based damping controller was synthesized [20]

This paper proposes a method of tuning the PID controller used for the DECS of a synchronous generator that is an extension of the well-established PID tuning method used in industry for the existing excitation control system, known as "cancelling zeros by poles." This method is modified to remove the unwanted effect of discretization on the controller performance by considering a suitable sampling time. The results show that when a discretized PID controller is tuned using the proposed method, it works well with a continuous plant and complies with the IEEE standard performance indexes. This design strategy also relieves the engineers and operators from the laborious task of calculating the PID gains while commissioning the new digital excitation system in the existing analog plant for upgrading.

The proposed method was tested on the IEEE standard simulation models to demonstrate the controller compatibility with the IEEE standard limit and was duly validated using Dspace 1104 industrial controller board hardware in the loop (HITL) that contains a continuous model of exciter and a brushless synchronous generator. 
The rest of the paper is organized as follows: Section 2 explains the IEEE standard excitation control system model. Section 3 explains the methods used for the PID controller tuning with the aim to comply with the IEEE standard performance indexes. Section 4 presents the application example with the simulation results and discussion. Section 5 explains the experimental work used to validate the proposed method and finally, the paper is concluded in Section 6.

\section{IEEE standard excitation control system model}

A "Type AC 5A" excitation control system model was used for the study and is shown in Fig 1. It is a simplified model for brushless excitation systems with a rotating rectifier.

The voltage regulator of this system is supplied from a source such as a permanent magnet generator, which benefits the regulator by not affecting its source voltage in case of disturbance. Unlike the other ac models, this model uses loaded rather than open circuit exciter saturation data in the same manner as that used for the dc models. This is a simplified model that is widely used in the industry because detailed data for the system is not required for such implementation.

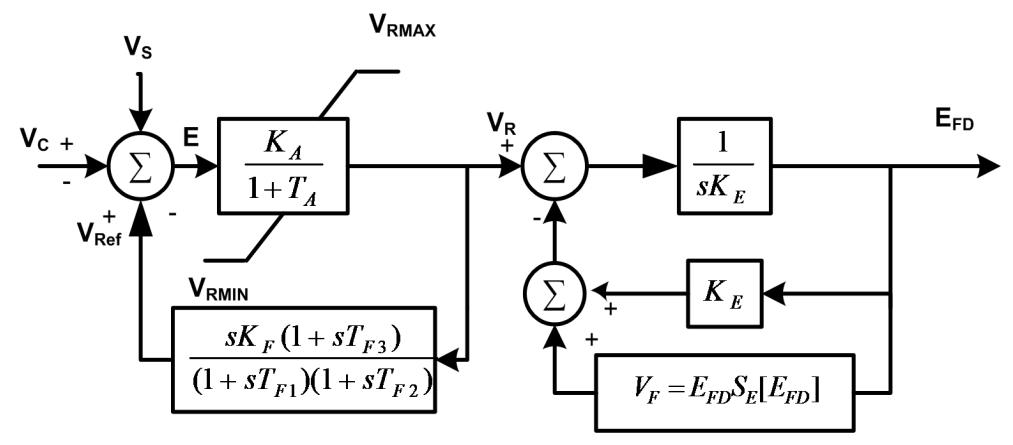

Fig. 1. The IEEE Type AC 5A model.

The model shown in Fig. 1 was modified to fit the PID controller and is shown in Fig 2. The design methodology proposed in this paper includes the calculation of the PID gains, i.e., the proportional gain $\left(\mathrm{K}_{\mathrm{P}}\right)$, integral gain $\left(\mathrm{K}_{\mathrm{I}}\right)$ and derivative gain $\left(\mathrm{K}_{\mathrm{D}}\right)$ using the transfer function of the standard model to facilitate this. The model in Fig. 2 is reduced to Fig. 3.

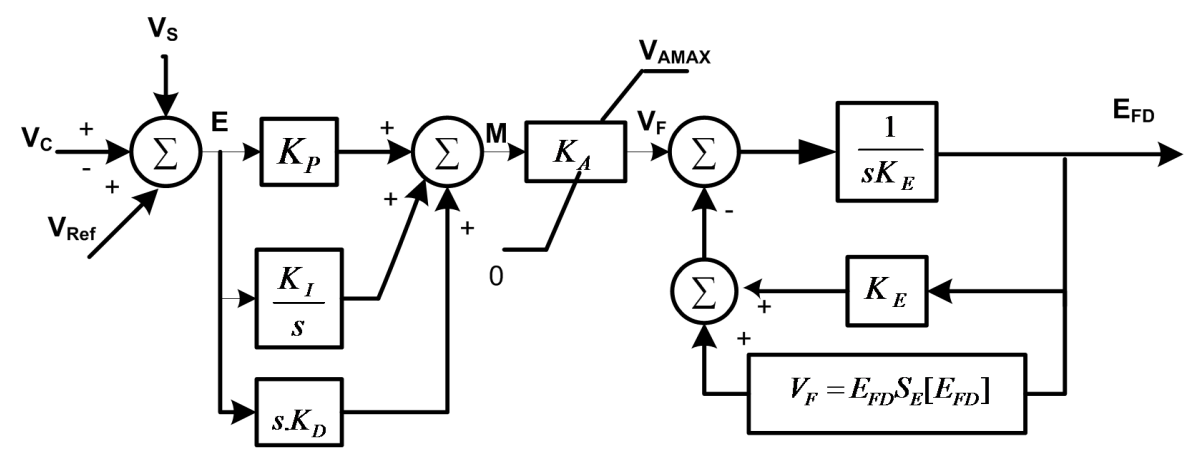

Fig. 2. The IEEE Type AC 5A model with a PID controller.

In the reduced model, the generators terminal voltage is the output, the generator and exciter blocks are reduced to the first order model. $\mathrm{Kg}$ is the forward gain of the generator, and tg and te are the generator and exciter time constants, respectively. The controller used to control the terminal voltage of the generator is a PID controller [2-3]. 


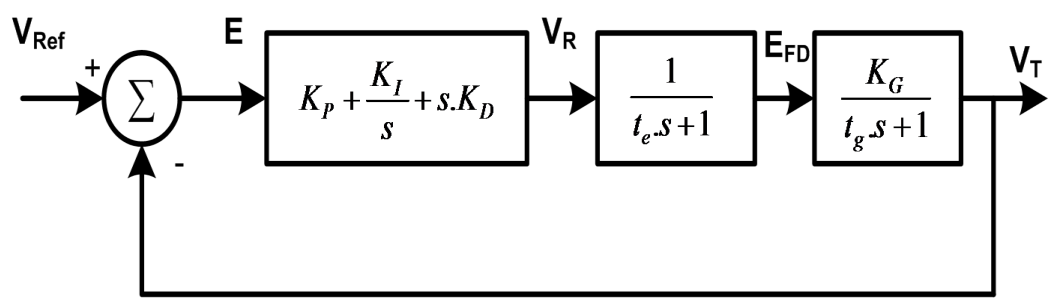

Fig. 3. The ECS reduced model.

The open-loop transfer functions of the generator, exciter, and the PID controller are $\mathrm{Gg}(\mathrm{s}), \mathrm{Ge}(\mathrm{s})$ and $\mathrm{Gc}(\mathrm{s})$, respectively and their relationship is given in (1), (2), and (3). The closed loop transfer function "TEC(s)" of the reduced model shown in Fig. 3 is given in (4).

$$
\begin{gathered}
G_{g}(s)=\frac{K_{G}}{t_{g} s+1} \\
G_{e}(s)=\frac{1}{t_{e} s+1} \\
G_{c}(s)=K_{P}+\frac{K_{I}}{s}+s K_{D} \\
T_{E C}(s)=\frac{G_{c}(s) \cdot G_{g}(s) \cdot G_{e}(s)}{1+G_{c}(s) \cdot G_{g}(s) \cdot G_{e}(s)}
\end{gathered}
$$

The PID controller Gc(s) needs to be tuned using the appropriate values of the gains, i.e., KP, KI, and $\mathrm{KD}$, in a manner such that the controller complies with the IEEE standard performance indexes.

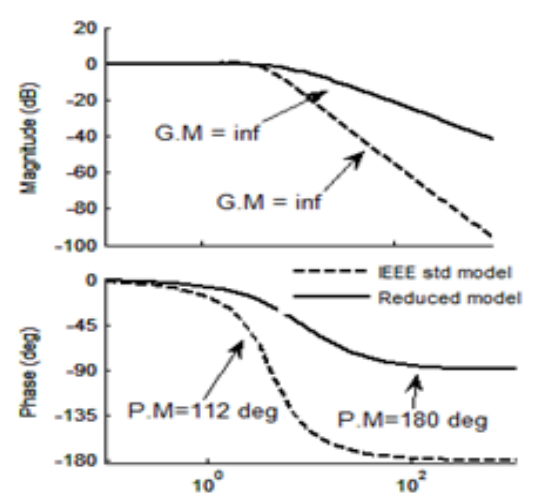

Fig. 4. The Bode response of the IEEE standard model and the reduced model of the excitation control system.

To ensure the design of the PID controller used for the excitation control system will work in the bounded region to maintain the dynamic stability using the reduced model, the bode response of the IEEE standard and reduced model of a typical excitation control system is given in Fig. 4 as a reference to fix the scale of the design in terms of the phase margin because a reduced model gives an increased phase margin when compared to the IEEE standard model. 


\section{PID controller design aim to comply IEEE standard.}

In the IEEE documents [4-7], guidelines are given in regard the identification, testing and evaluation of the dynamic performance of the excitation control system and accordingly, the accepted values of the performance indexes characterizing a good feedback control system performance are given in Table 1 . The values reported in this table are for those generators that are not connected to the loads.

Table 1. The IEEE standard performance index.

\begin{tabular}{cc}
\hline Index & Value \\
\hline Overshoot & $\leq 20 \%$ \\
Gain Margin & $\geq 10 \mathrm{db}$ \\
Phase Margin & $\geq 40^{\circ}$ \\
\hline
\end{tabular}

The range of the performance indexes of the excitation control system with small signal dynamics are given in Table 2 . The values reported in this table vary in the specified range on the basis of the capacity of the generators and their loading conditions.

To achieve good performance indexes for the controller, as specified in the standard, the controller design requires an appropriate tuning of the tepid controller. The tuning strategies used for analog PID controllers currently used in the industry are the pole placement method (direct design approach) and the pole zero cancellation method. Both have pros and cons in terms of the laborious mathematical calculation and flexibility in the custom design. These methods are popular in the industry owing to their capability of converging to a solution even when the precise data for the synchronous generator is not available [3]. This paper considers cancelling zeros using the poles method, which ensures quick design and excludes the mathematical complexities by avoiding trial and error, as presented in subsection $\mathrm{A}$. The proposed method used to tune the discrete PID controller is presented in subsection B. In this method, a previously selected analog method was extended with modification to moderate the effect of discretization by considering a suitable sampling time. To support the applicability of the modification to the design, a fact was considered in the method, i.e., 'a discrete PID controller and a continuous PID controller behave the same when the sampling time chosen is very small'.

Table 2. The IEEE standard performance index with a small signal dynamic.

\begin{tabular}{cc}
\hline Index & Value \\
\hline Rise time & $\leq 1 \mathrm{~s}$ \\
Settling time & $\leq 4 \mathrm{~s}$ \\
Bandwidth & 0.3 to $12 \mathrm{~Hz}$ \\
\hline
\end{tabular}

\subsection{Analog PID controller design}

The method selected for the analog PID controller design is "cancelling zeros by poles." Academically, this method is impractical, but in the rotary excitation system, the poles corresponding to the generator and the exciter lie far away from each other (i.e., exciter poles lying 6 to 10 times away from the generator pole) in the pole zero map as reported in [3]. When the loop gain increases, the pole moves toward the corresponding zeros. Since the poles of the exciter and the generator are well separated, the resulting system through the cancellation of zeros by the poles does not affect the original systems 
required dynamics. This makes the selected method suitable for the rotary excitation system application [3].

The open-loop system transfer function of the reduced model is shown in Fig. 3 with a typical assumption of $\mathrm{Kg}=1$ and given in (5).

$$
G_{c}(s) G(s)=\frac{K_{D}\left(s^{2}+\frac{K_{P}}{K_{D}} s+\frac{K_{I}}{K_{D}}\right)}{t_{g} t_{e} s\left(s+\frac{1}{t_{g}}\right)\left(s+\frac{1}{t_{e}}\right)}
$$

The numerator and the denominator of the transfer function (5) have two zeros and three poles, and the cancellation of two zeros with two farthest poles of (5) is achieved by ensuring the equation in (6).

$$
\frac{\left(s^{2}+\frac{K_{P}}{K_{D}} s+\frac{K_{I}}{K_{D}}\right)}{\left(s+\frac{1}{t_{g}}\right)\left(s+\frac{1}{t_{e}}\right)}=1
$$

Equating the numerator and denominator of (6) results in (7).

$$
s^{2}+\frac{K_{P}}{K_{D}} s+\frac{K_{I}}{K_{D}}=\left(s+\frac{1}{t_{g}}\right)\left(s+\frac{1}{t_{e}}\right)
$$

Expansion and arrangement makes the RHS polynomial of (7) as in (8).

$$
s^{2}+\frac{K_{P}}{K_{D}} s+\frac{K_{I}}{K_{D}}=s^{2}+\left(\frac{t_{g}+t_{e}}{t_{g} t_{e}}\right) s+\frac{1}{t_{g} t_{e}}
$$

Equating the LHS and RHS of equation (8) gives an expression for KP in (9) and KI in (10).

$$
\begin{gathered}
K_{P}=K_{D}\left(\frac{t_{g}+t_{e}}{t_{g} t_{e}}\right) \\
K_{I}=\frac{K_{D}}{t_{g} t_{e}}
\end{gathered}
$$

Submitting KP and KI in (5) results in (11).

$$
G_{c}(s) \cdot G(s)=\frac{K_{D}}{t_{g} t_{e} s}
$$

The closed loop transfer function of the system in Fig. 3 using (11) as in (12).

$$
\frac{G_{c}(s) G(s)}{1+G_{c}(s) G(s)}=\frac{K_{D} / t_{g} t_{e} s}{1+K_{D} / t_{g} t_{e} s}
$$

The time response of the system in (12) to a unit step input is given in (13). 


$$
C(\mathrm{t})=1-\mathrm{e}^{-\frac{\mathrm{K}_{\mathrm{D}}}{\mathrm{t}_{\mathrm{g}} \mathrm{e}}}
$$

If " $\mathrm{t}_{\mathrm{r}}$ " is the required rise time for the design, it is defined as the time required for the response to rise from $10 \%$ to $90 \%$ of its final value and the resulting expression for $\mathrm{K}_{\mathrm{D}}$ is given by (14).

$$
K_{D}=\frac{t_{g} t_{e}}{t_{r} K_{G}} \cdot \ln 9
$$

Derivative gain KD in (14) depends on the plant parameters tg, te, and the desired rise time "tr." An observation shows that the controller design is aims for a faster response with the proposed method making the settling time (ts), percentage peak overshoot (\%MP), gain margin (G.M) and phase margin (P.M) comply with the IEEE standard range, as shown in Table 1 and Table 2.

\subsection{The design of the digital PID controller}

The digital PID controller is implemented on a computer chip by the discretization of an integral and derivative block, and the computer chip reads the continuous error signal at regular time intervals, which is referred to as the sampling time.

In the proposed tuning method used for the discrete PID controller, the analog gains calculated earlier in subsection 3.1 were used with an assumption that the behavior of the discrete PID controller is similar to the continuous PID controller when the sampling time is very small.

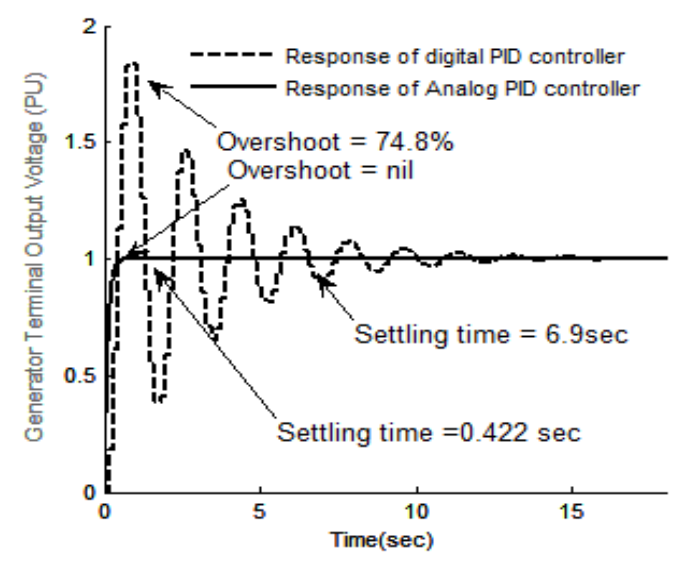

Fig. 5. A comparison between the typical analog and the digital ECS's step response. The discrete controller was tuned using a non-modified analog gain.

$$
G_{c}(z)=K_{P}+\frac{T\left(1+z^{-1}\right)}{2 .\left(1-z^{-1}\right)} K_{I}+\frac{\left(1-z^{-1}\right)}{T} K_{D}
$$

However, if the gains calculated using the existing method described earlier are directly used to implement the discrete PID controller, the system becomes unstable as described by the step and bode response of a typical excitation control system in Figs. 5 and 6.

The proposed method aims to tune the discrete PID controller with a large sampling time with a limit of being two times shorter than the lowest exciter time constant such that the design complies with the IEEE standard. 
The existing PID controller in (3) is discretized by replacing the integral term " $1 / \mathrm{s}$ " with " $\mathrm{T}(1+\mathrm{z}-$ $1) / 2(1-z-1)$ " using trapezoidal integration and a derivative term "s" with $(1-z-1) / T$, where $T$ is the sampling time. Thus, the discretized controller equation is shown in (15).

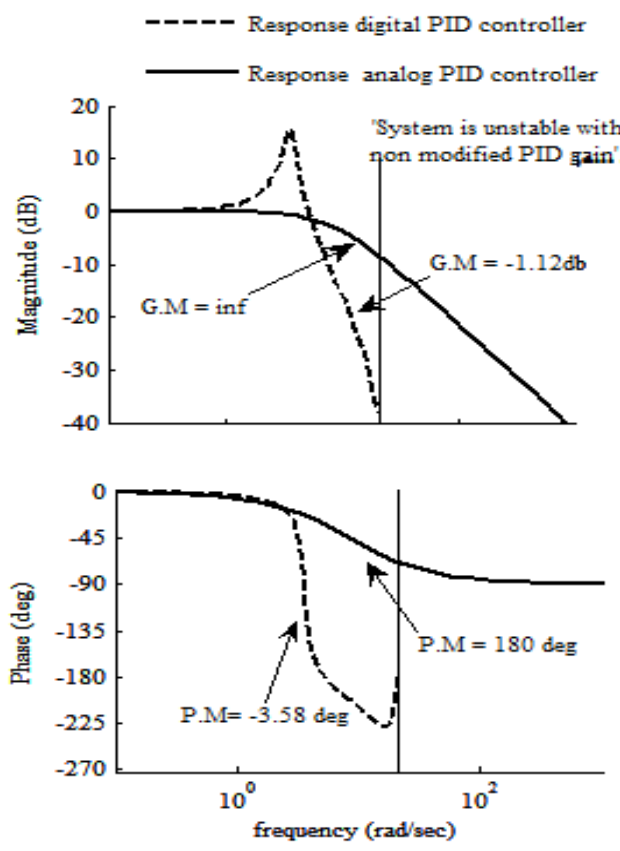

Fig. 6. A comparison between the typical analog and the digital ECS's bode response. The discrete controller was tuned using a non- modified analog gain.

The second term on the RHS of (15) is rearranged in (16).

$$
\begin{aligned}
G_{c}(z)= & K_{P}+\frac{K_{I} T}{\left(1-z^{-1}\right)}+\frac{K_{I} T\left(Z^{-1}-1\right)}{2 \cdot\left(1-z^{-1}\right)}+ \\
& +\frac{\left(1-z^{-1}\right)}{T} K_{D}
\end{aligned}
$$

Further arrangement of (16) results in (17).

$$
G_{c}(z)=K_{P}-\frac{K_{I} T}{2}+\frac{K_{I} T}{\left(1-z^{-1}\right)}+\frac{\left(1-z^{-1}\right)}{T} K_{D}
$$

The RHS of equation (17) is arranged to $\mathrm{z}$ variable terms with coefficients $\mathrm{K}_{\mathrm{PD}}, \mathrm{K}_{\mathrm{ID}}$ and $\mathrm{K}_{\mathrm{DD}}$ as shown in (18).

$$
\left.\begin{array}{c}
G_{c}(z)=K_{P D}+\frac{K_{I D}}{\left(1-z^{-1}\right)}+K_{D D}\left(1-z^{-1}\right) \\
K_{P D}=K_{P}-\frac{K_{I} T}{2} \\
K_{I D}=K_{I}^{T} \\
K_{D D}=\frac{K_{D}}{T}
\end{array}\right\}
$$

Equating the RHS of (17) and (18) gives discrete PID gains $\mathrm{K}_{\mathrm{PD}}, \mathrm{K}_{\mathrm{ID}}$, and $\mathrm{K}_{\mathrm{DD}}$ in (19) in terms of 
analog PID gains $K_{P}, K_{I}, K_{D}$ and sampling time ' $T$ '.

The block diagram in Fig. 7 approximates the DECS where the discrete PID controller block interacts with a continuous exciter and a generator block to a continuous model. Therefore, the analysis of this system was performed by linearizing the discrete controller block. The analog PID gains $K_{P}, K_{I}$ and $K_{D}$ were calculated using equations (9), (10), and (14), and the resulting analog PID gains were used in equation (19) to calculate the discrete PID gains $K_{P D}, K_{I D}$, and $K_{D D}$. It is observed that with the adjustment of the forward loop gain, the " $\mathrm{K}_{\mathrm{G}}$ "compatibility of the controller with the IEEE standard and, consequently, the stability of the system in Fig. 7 is easily ensured.

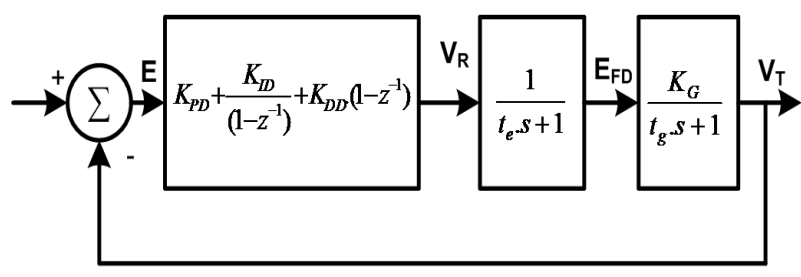

Fig. 7. The approximated block diagram of a digital ECS.

\section{Results and Discussion}

The rotary excitation system of $500 \mathrm{KVA}, 440 \mathrm{~V}$, and a $50 \mathrm{~Hz}$ brushless synchronous generator, whose manufacturer's data are reported in Table 3, was used to demonstrate the methods described in Section 3.

Table 3. The parameters of the brushless synchronous generator used in this study.

\begin{tabular}{cc}
\hline Generator time constant "tg" & $2.2 \mathrm{~s}$ \\
\hline Moment of Inertia & $8.0068 \mathrm{Kg} \cdot \mathrm{m}^{2}$ \\
\hline Damping factor & 65 \\
\hline No of poles & 2 \\
\hline $\mathrm{R}$ & $0.0049 \Omega$ \\
\hline $\mathrm{L}$ & $0.1273 \times 10^{-3} \mathrm{H}$ \\
\hline
\end{tabular}

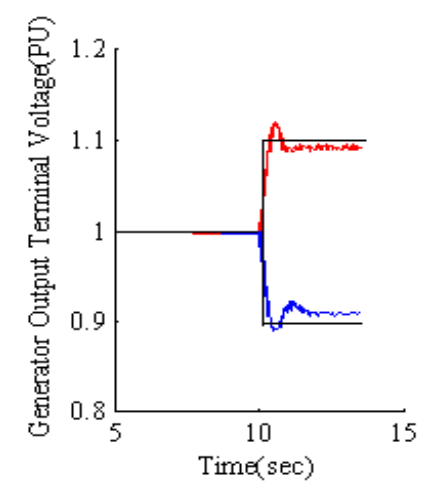

Fig. 8. The $10 \%$ step increment and decrement response of the generator terminal voltage when the AVR consists of the proportional controller only.

The solution for this problem is the use of a PI controller or PID controller. The disadvantage of the PI controller is the integral gain that decreases the system's dynamics and increases the phase delay and consequently decreases the system's stability. The proposed controller used for this system is the PID controller whose derivative element compensates the system's deceleration caused by an integral element.

The excitation system is an analog type and provided with an automatic volatage regulator (AVR) that 
has a proportional "P" controller in the forward path. This generator's output terminal voltage (PU) response for a $10 \%$ step increment and a $10 \%$ step decrement of the reference voltage is given in Fig. 8 and confirms the terminal voltage does not reach a steady state and consequently makes the power system unstable.

The data sheet provided by the manufacturer does not contain the value of the exiter time constant; therefore, for the design of the controller, the exciter time constant was approximated as one sixth of the generator time constant, as proposed in [3].

\subsection{Example: Analog PID controller}

The calculation of the PID gain for the controller to be used in the excitation system of the generator in Section 4 uses equation (9), (10), and (14) with a desired rise time (tr) of $0.236 \mathrm{~s}$. The calculated PID gains are $\mathrm{KP}=23, \mathrm{KI}=9$, and $\mathrm{KD}=7$. Thus, the closed loop transfer function of the system in (4) is given in (20).

$$
T_{E C}(s)=\frac{\left(7 s^{2}+23 s+9\right)}{0.8067 s^{3}+9.567 s^{2}+24 s+9}
$$

The step and bode response of the reduced system model in (20) was compared with the IEEE standard model (Fig. 2) and is shown in Figs. 9 and 10, respectively.

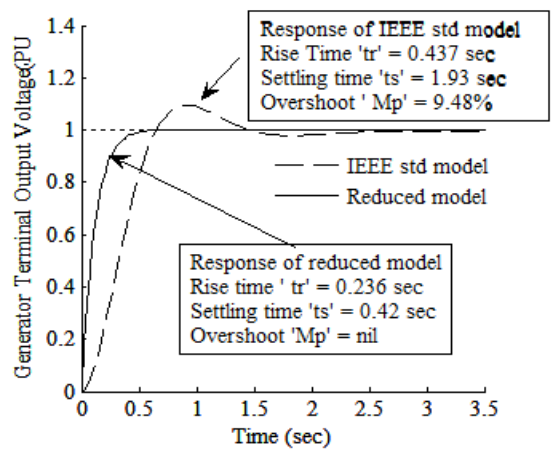

Fig. 9. A comparison of the reduced and IEEE standard model's step response for the analog PID controller.
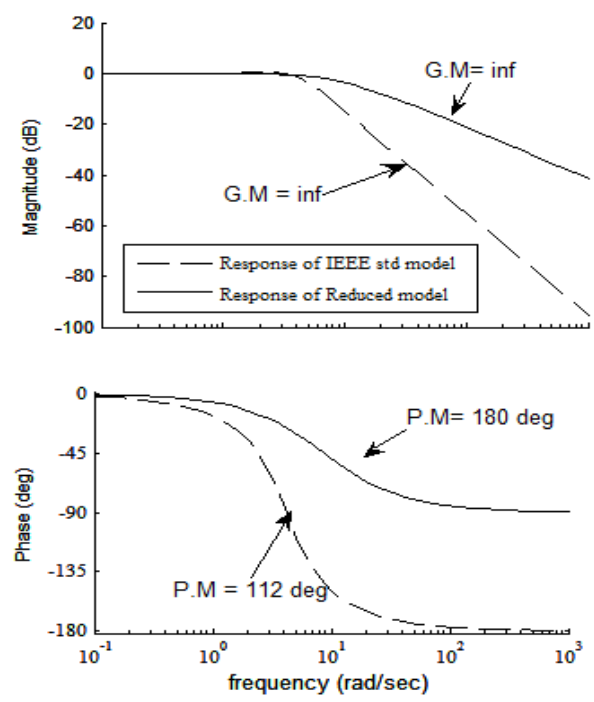

Fig. 10. A comparison of the reduced and IEEE standard model's bode response for the analog PID controller. 


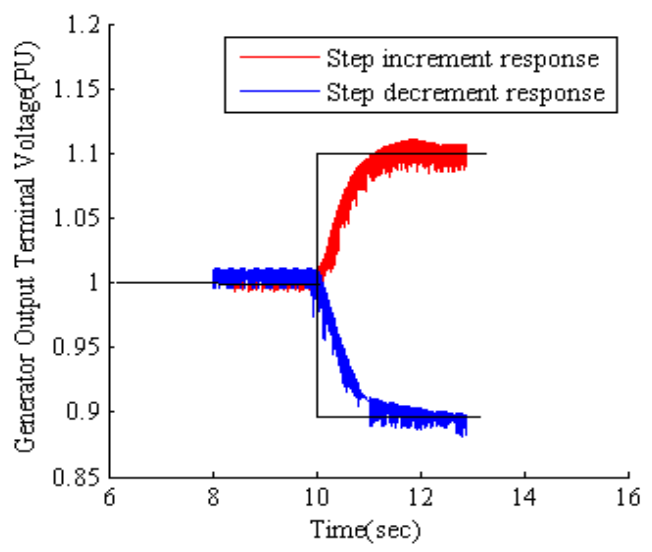

Fig. 11. The 10\% step increment and decrement voltage response of the capacitive loaded generator.

It can be seen that the method ensures the compatibility of an analog PID controller with the IEEE standard performance indexes, as reported in Tables 1 and 2. The performance of the controller was further investigated by connecting it to a loaded generator and the load connected to the generator was capacitive.

The generator terminal voltage output response (PU) for a $10 \%$ step increment and $10 \%$ step decrement to the reference voltage is shown in Fig 11, and the response for a $100 \%$ load connection at $\mathrm{t}=$ $12 \mathrm{~s}$ and rejection at $\mathrm{t}=13 \mathrm{~s}$ is shown in Fig. 12, which confirms the stable and an improved operation of the generator with the controller.

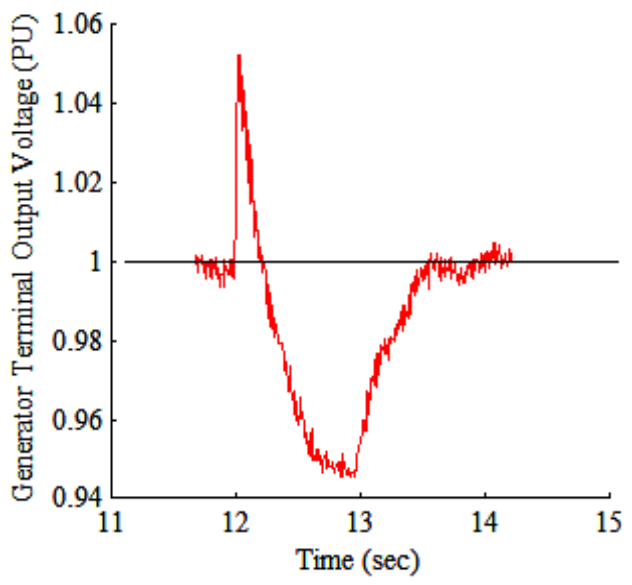

Fig. 12. The connection of load for $100 \%$ transient from offline to online.

\subsection{Example: Digital PID controller}

The analog PID controller gains described in the previous section were used to calculate the gains of the digital PID controller KPP, KID, and KDD using equation (19) described in subsection B of Section 3; a sampling time of $\mathrm{T}=0.15 \mathrm{~s}$ was used, which is two times shorter than the lowest exciter time constant. The analog gains KP, KI, and KD were multiplied by 10 to account for the other gain in the regulator. The digital PID controller gains calculated are KPP $=223, \mathrm{KID}=13$, and $\mathrm{KDD}=466$.

Then, the design was tested on the simulation model of the standard IEEE excitation system 421.5 Type AC-5A as shown in Fig. 3 by considering the anti-reset and wind-up for integral action. 


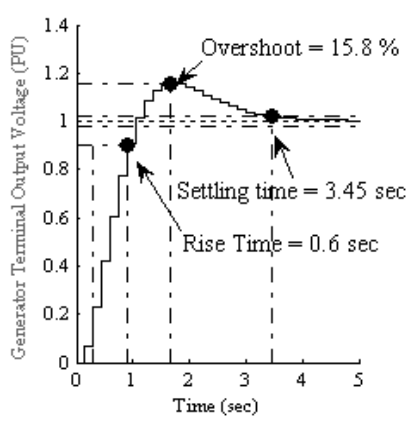

Fig. 13. The unit step response of the digital excitation control system (DECS).
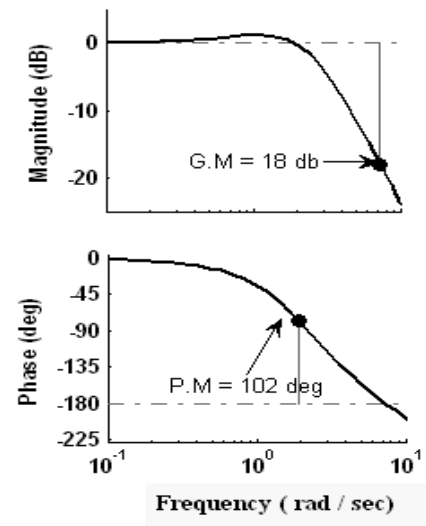

Fig. 14. The Bode response of the DECS.

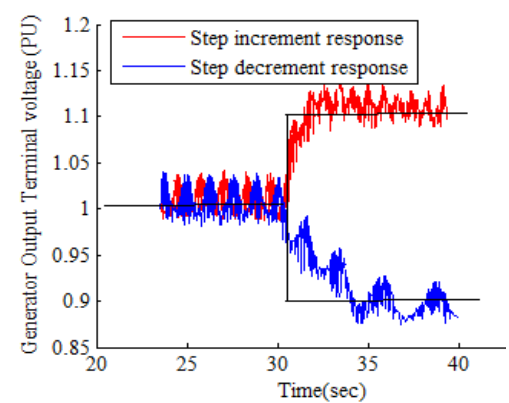

Fig. 15. The $10 \%$ step increment and decrement voltage response of the generator with a capacitive load.

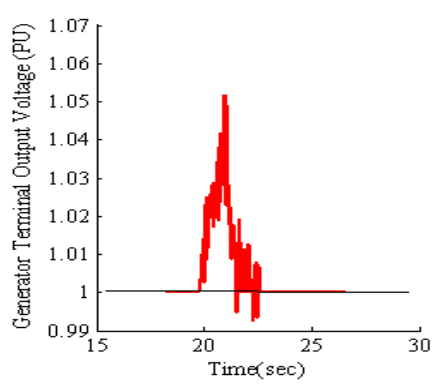

Fig. 16. Load rejection: $100 \%$ Transient for online to offline transition. 
The step and bode response of the designed controller used for the DECS after adjusting the value of the forward gain KG to 0.045 is shown in Fig. 13 and 14, which show a rise time of $0.6 \mathrm{~s}$, overshoot of $15.8 \%$, settling time of 3.45 s, G.M of $18 \mathrm{db}$ and P.M of $102^{\circ}$. The values of the performance indexes comply with the IEEE standard and thus, the stability of the system is ensured

The performance of the digital PID controller was further investigated by connecting it to a generator with a capacitive load. The generator terminal voltage output response (PU) for a $10 \%$ step increment and $10 \%$ step decrement to the reference voltage is given in Fig. 15 and the response when a $100 \%$ load was rejected at $\mathrm{t}=20 \mathrm{~s}$ and reconnected at $\mathrm{t}=22 \mathrm{~s}$ is shown in Fig. 16.

\section{Experimentation Using DS 1104 Hardware}

The DS1104 controller board is real-time hardware based on PowerPC technology, and its set of I/O interfaces allows the controller board to be used for developing controllers for various systems with a real-time interface (RTI). The function models on the DS1104 controller board can be easily configured and run by inserting the blocks into a simulink block diagram and generating the model code via a simulink coder.

The real-time model is compiled, downloaded, and started automatically in the MATLAB platform. The experimental setup for the HITL experiment used to test the designed digital PID controller in the excitation control system is given in Fig. 17. The discretized PID controller was built in a Dspace 1104 controller board using a RTI with the MATLAB simulink and the continuous model of the plant (i.e., the exciter and the generator) was built in the PC.

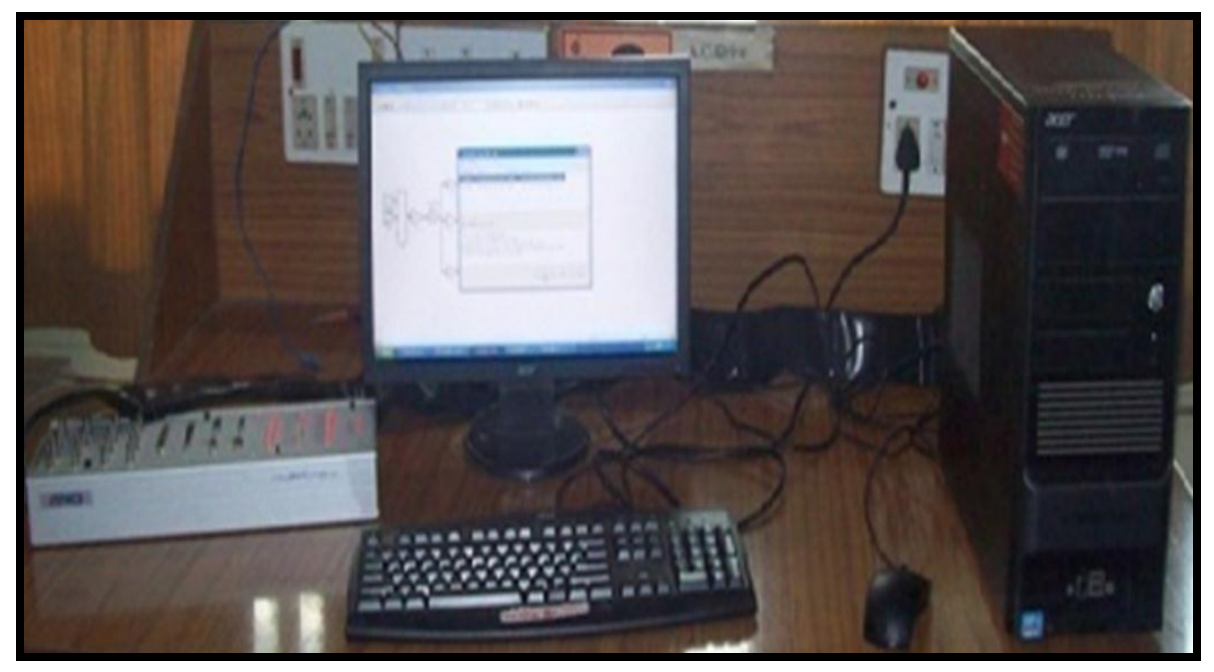

Fig. 17. The experimental setup with DS 1104 in the loop to test the designed DECS.

Thus, the scheme takes the shape of hardware in the loop (HITL) and follows the IEEE standard model given in Fig. 2. When the digital PID controller is used in the excitation system of $500 \mathrm{KVA}, 440 \mathrm{~V}$, and a $50 \mathrm{~Hz}$ synchronous generator, the time response of this generator is shown in Fig. 18 wherein, the terminal voltage reaches a steady state at $t=8 \mathrm{~s}$ and the stability of the system is ensured.

This experiment validates the method used for the design of the DECS. A comparison of the performance of the PID controller with gains calculated using the proposed modified method and the nonmodified gains calculated using the cancelling poles by zeros method demonstrates the improvement in the performance of the controller with the modified method, as reported in Table 4. 


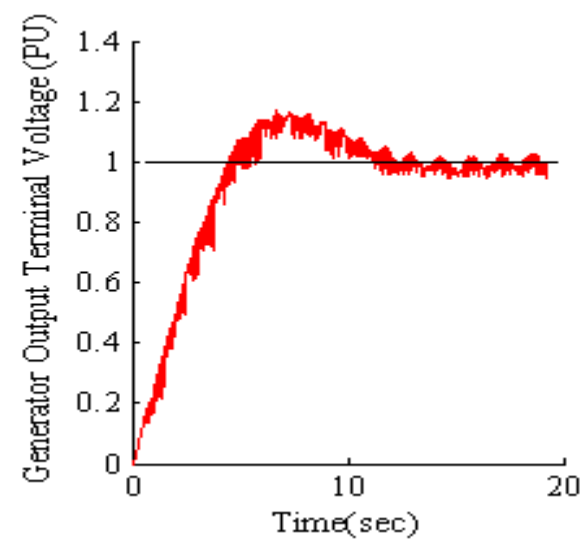

Fig. 18. The generator output terminal voltage using the DECS.

Table 4. A comparison of the PID controller performance.

\begin{tabular}{ccc}
\hline $\begin{array}{c}\text { Performance } \\
\text { Index }\end{array}$ & $\begin{array}{c}\text { Non-modified } \\
\text { PID gains } \mathrm{K}_{\mathrm{P}}= \\
23, \mathrm{~K}_{\mathrm{I}}=9 \text { and } \mathrm{K}_{\mathrm{D}} \\
=7\end{array}$ & $\begin{array}{c}\text { Modified PID } \\
\text { gains } \mathrm{K}_{\mathrm{PD}}=223, \\
\mathrm{~K}_{\mathrm{ID}}=13 \text { and } \mathrm{K}_{\mathrm{DD}} \\
=466\end{array}$ \\
\hline Rise Time (s) & 0.36 & 0.6 \\
\hline Settling Time (s) & 6.9 & 3.45 \\
\hline Overshoot (\%) & 74.8 & 15.8 \\
\hline G.M (db) & -1.12 & 18 \\
\hline Phase Margin $\left({ }^{\circ}\right)$ & -3.58 & 102 \\
\hline
\end{tabular}

\section{Conclusions}

A PID controller has been designed using the proposed method to ensure the terminal voltage of a synchronous generator at the rated value. The use of the existing PID gains in the proposed modified method makes the design process not only simple but also ensures that the design of the discrete PID controller is IEEE compliant. The designed PID controller used in the AVR of a digital excitation system was tested and validated using the HITL technique. An investigation shows that the sampling time for the design may not necessarily be very small; thus, expensive processors can be avoided. However, the drawback of this method is that the customized and a competitive design of the digital PID controller cannot be ensured for the excitation system owing to the complexity involved in the design. This complexity is a result of the conversion of the discrete PID controller, sample and hold block into an s domain using the Pade approximation, which makes the order of the closed loop transfer function very high. This work can further be extended for a competitive and customized design of the digital PID controller with the aim of automating the tuning process of the PID controller in the digital excitation system.

\section{References}

[1] Andrea B, Federico D, Marco I, Adriano P, Renato P. A feedback linearization scheme for the control of synchronous generators. Electric Power Components and Systems. 2012; 40(16): 1842-1869. 
[2] Godhwani, Basler MJ. A digital excitation control system for use on brushless excited synchronous generators. IEEE Transactions on Energy Conversion. 1996; 11(3): 616-620.

[3] Kiyong K, Richard CS. Tuning a PID controller for a digital excitation control system. IEEE Transactions on Industry Applications. 2005; 41(2): 485-492.

[4] IEEE Guide for Identification, Testing, and Evaluation of the Dynamic Performance of Excitation Control Systems, IEEE STD 421.2, 2014.

[5] IEEE Recommended Practice for Excitation System Models for Power System Stability Studies, IEEE STD 421.5, 2005.

[6] IEEE Standard Definitions for Excitation Systems for Synchronous Machines, IEEE STD 421.1, 2007.

[7] IEEE Guide for Preparation of Specification for Excitation Systems, IEEE STD 421.4, 1990.

[8] Abul RH, Sadrul Ula AHM. Design and implementation of a fuzzy controller based automatic voltage regulator for a synchronous generator. IEEE Transactions on Energy Conversion. 1994; 9(3): 550-557.

[9] Mahit G, Necati D. Fuzzy control of brushless excitation system for steam turbogenerators. IEEE Transactions on Energy Conversion, 2010; 25(3): 844-852.

[10] Longquan X, Jianhua W, Cong P. Backstepping control of digital excitation systems based on neural network. IEEE International Conference on Industrial Technology. 2008; 1-5.

[11] Saptarshi D, Indranil P. On the mixed $\mathrm{H} 2 / \mathrm{H} \infty$ loop shaping trade-offs in fractional order control of the AVR system. IEEE Transactions on Industrial Informatics, 2014; 10(4): 1982-1991.

[12] Jiang H, Yin X, Chen Y, Li D. Optimal excitation control of synchronous generator based on adaptive ants colony algorithm. Third International Conference on Natural Computation, 2007, IEEE. 3: 3-7.

[13] Chuan Y, Ganesh KV, Keith C. AIS-Based coordinated and adaptive control of generator excitation systems for an electric ship. IEEE Transactions on Industrial Electronics. 2012; 59(8): 3102-3112.

[14] Saavedra-Montes AJ, Ramos-Paja CA, Orozco-Gutierrez ML, Cifuentes WA, Ramirez-Scarpetta JM. Calculation of excitation system controllers to fulfill IEEE standard performance indexes. Electric Power Systems Research, 2012; 89: 196-203.

[15] Kiyong K, Pranesh R, Jeffrey AB. Self-Tuning of the PID controller for a digital excitation control system. IEEE Transactions On Industry Applications, 2010; 46(4): 1518-1524.

[16] Konara KMSY, Kolhe ML. Pitch controller modeling for wind turbine power regulation using feed forward control strategies. In: Proc. of Power and Energy Engineering Conference (APPEEC), IEEE PES Asia-Pacific. 2015; 1-5.

[17] Patidar NP, Kolhe ML, Tripathy NP, Sahu B, Sharma A, Nagar LK, Azmi AN. Optimized design of wide-area PSS for damping of inter-area oscillations. In: Proc. of IEEE 11th International Conference on Power Electronics and Drive Systems.2015; 1172-1177.

[18] Panda S, Patidar NP, Mohan K. Cascaded PD-PI controller for active power frequency control of two-area multi-units power system. IEEE International Conference Power and Renewable Energy (ICPRE), 2016.

[19] Michal L, Miroslaw W. Sampling rate impact on the tuning of PID controller parameters. Intl Journal of Electronics and Telecommunications. 2016; 62(1).

[20] Akshay S, Nagar LK, Patidar NP, Kolhe ML, Nandanwar SR, Puranik VN, Singh VK. Minimizing uncertainties with improved power system stability using wide area fuzzy-2 logic based damping controller. In: Proc. of IEEE 3rd International Conference on Computational Intelligence \& Communication Technology (CICT), 2017; 1-5. 